\title{
Boundedness of sublinear operators in Triebel-Lizorkin spaces via atoms
}

\author{
by
}

\author{
LiguANG LiU and DACHUN YANG (Beijing)
}

\begin{abstract}
Let $s \in \mathbb{R}, p \in(0,1]$ and $q \in[p, \infty)$. It is proved that a sublinear operator $T$ uniquely extends to a bounded sublinear operator from the Triebel-Lizorkin space $\dot{F}_{p, q}^{s}\left(\mathbb{R}^{n}\right)$ to a quasi-Banach space $\mathcal{B}$ if and only if

$\sup \left\{\|T(a)\|_{\mathcal{B}}: a\right.$ is an infinitely differentiable $(p, q, s)$-atom of $\left.\dot{F}_{p, q}^{s}\left(\mathbb{R}^{n}\right)\right\}<\infty$, where the $(p, q, s)$-atom of $\dot{F}_{p, q}^{s}\left(\mathbb{R}^{n}\right)$ is as defined by Han, Paluszyński and Weiss.
\end{abstract}

1. Introduction. It is known that atomic characterization is a powerful tool in investigating the boundedness of operators in Hardy spaces on Euclidean spaces. In principle, boundedness of operators in Hardy spaces can be deduced from their behavior on atoms. However, Meyer, Taibleson and Weiss [21, p. 513] gave an example of $f \in H^{1}\left(\mathbb{R}^{n}\right)$ whose norm cannot be attained by finite decompositions of into $(1, \infty)$-atoms; see also $[12,2]$. Based on this fact, Bownik [2, Theorem 2] constructed a linear functional defined on a dense subspace of $H^{1}\left(\mathbb{R}^{n}\right)$, which maps all $(1, \infty)$-atoms into bounded scalars, but does not extend to a bounded linear functional on the whole $H^{1}\left(\mathbb{R}^{n}\right)$. This implies that proving that a (sub)linear operator $T$ maps all $(p, \infty)$-atoms into uniformly bounded elements of $\mathcal{B}$ cannot guarantee the boundedness of $T$ from the whole $H^{p}\left(\mathbb{R}^{n}\right)$ with $p \in(0,1]$ to some quasi-Banach space $\mathcal{B}$. This phenomenon was also essentially observed by Meyer and Coifman in [20, p. 19].

Then a natural question is to find some simple and useful conditions associated with atoms which can guarantee the boundedness of (sub)linear

2000 Mathematics Subject Classification: Primary 46E35; Secondary 42B20, 42B25, $47 \mathrm{~A} 30$.

Key words and phrases: Triebel-Lizorkin space, Lusin area function, atom, sublinear operator, quasi-Banach space.

The second author (who is the corresponding author) is supported by the National Science Foundation for Distinguished Young Scholars (No. 10425106) of China. Both authors would like to thank the referee and the copy editor, Jerzy Trzeciak, for their valuable remarks which made this article more readable. 
operators in Hardy spaces. In fact, Yabuta [24] gave some sufficient conditions for the boundedness of linear operator $T$ from $H^{p}\left(\mathbb{R}^{n}\right)$ with $p \in(0,1]$ to $L^{q}\left(\mathbb{R}^{n}\right)$ with $q \geq 1$ or $H^{q}\left(\mathbb{R}^{n}\right)$ with $q \in[p, 1]$. By means of the LittlewoodPaley $S$-function characterization of $H^{p}\left(\mathbb{R}^{n}\right)$, it was proved in [25] that a sublinear operator $T$ extends to a bounded sublinear operator from $H^{p}\left(\mathbb{R}^{n}\right)$ with $p \in(0,1]$ to some quasi-Banach space $\mathcal{B}$ if and only if $T$ maps all $(p, 2, s)$-atoms of $H^{p}\left(\mathbb{R}^{n}\right)$ for some $s \geq\lfloor n(1 / p-1)\rfloor$ into uniformly bounded elements of $\mathcal{B}$; here and in what follows, $\lfloor x\rfloor$ for $x \in \mathbb{R}$ denotes the maximal integer no more than $x$. Using the Calderón reproducing formula, Zhao [27] independently proved that if $T$ is a linear operator which is bounded on $L^{2}\left(\mathbb{R}^{n}\right)$ and also uniformly bounded on all $\left(p, 2, s_{0}\right)$-atoms of $H^{p}\left(\mathbb{R}^{n}\right)$ with $s_{0}=\lfloor n(1 / p-1)\rfloor$, then $T$ extends to a bounded linear operator from $H^{p}\left(\mathbb{R}^{n}\right)$ to $L^{p}\left(\mathbb{R}^{n}\right)$. We mention that the result of Yabuta [24] was generalized to Ahlfors 1-regular metric measure spaces in [17], and the result of [25] was extended to RD-spaces in [26], where an RD-space (see $[14,15]$ ) is a space of homogeneous type in the sense of Coifman and Weiss $[7,8]$ with the additional property that a reverse doubling property holds. Comparing these results with the example of Meyer, Taibleson and Weiss in [21] and Bownik's results in [2], we see that as regards the boundedness of sublinear operators in Hardy spaces, there exists a structural difference between $(p, 2, s)$-atoms and $(p, \infty, s)$-atoms.

Recently, Meda, Sjögren and Vallarino [19] independently obtained a remarkable result by a different method from [25]. For $q \in(1, \infty]$, denote by $H_{\text {fin }}^{1, q}\left(\mathbb{R}^{n}\right)$ the vector space of all finite linear combinations of $(1, q, 0)$-atoms of $H^{1}\left(\mathbb{R}^{n}\right)$ endowed with the norm

$$
\begin{aligned}
&\|f\|_{H_{\text {fin }}^{1, q}\left(\mathbb{R}^{n}\right)} \equiv \inf \left\{\sum_{j=1}^{N}\left|\lambda_{j}\right|: f=\sum_{j=1}^{N} \lambda_{j} a_{j}, N \in \mathbb{N},\left\{\lambda_{j}\right\}_{j=1}^{N} \subset \mathbb{C}\right. \text {, and } \\
&\left.\left\{a_{j}\right\}_{j=1}^{N} \text { are }(1, q, 0) \text {-atoms of } H^{1}\left(\mathbb{R}^{n}\right)\right\} .
\end{aligned}
$$

By means of the grand maximal function characterization for $H^{1}\left(\mathbb{R}^{n}\right)$, Meda, Sjögren and Vallarino [19] proved that $\|\cdot\|_{H^{1}\left(\mathbb{R}^{n}\right)}$ and $\|\cdot\|_{H_{\text {fin }}^{1, q}\left(\mathbb{R}^{n}\right)}$ are equivalent quasi-norms on $H_{\text {fin }}^{1, q}\left(\mathbb{R}^{n}\right)$ with $q \in(1, \infty)$ or on $H_{\text {fin }}^{1, \infty}\left(\mathbb{R}^{n}\right) \cap$ $\mathcal{C}\left(\mathbb{R}^{n}\right)$, where $\mathcal{C}\left(\mathbb{R}^{n}\right)$ denotes the set of continuous functions. From this, they further deduced that a linear operator defined on $H_{\text {fin }}^{1, q}\left(\mathbb{R}^{n}\right)$ which maps $(1, q, 0)$-atoms of $H^{1}\left(\mathbb{R}^{n}\right)$ or continuous $(1, \infty, 0)$-atoms of $H^{1}\left(\mathbb{R}^{n}\right)$ into uniformly bounded elements of some Banach space $\mathcal{B}$ uniquely extends to a bounded operator from $H^{1}\left(\mathbb{R}^{n}\right)$ to $\mathcal{B}$. In [13], the full results of [19] are generalized to $H^{p}(\mathcal{X})$ and quasi-Banach-valued sublinear operators, where $\mathcal{X}$ is an $\mathrm{RD}$-space having "dimension $n$ " in some sense and $p \in(n /(n+1), 1]$. 
It is also well-known that Triebel-Lizorkin spaces embrace many classical function spaces, such as Lebesgue spaces, Hardy spaces, BMO and Sobolev spaces; see [23]. Frazier and Jawerth established a "smooth" atomic decomposition for the Triebel-Lizorkin spaces based on the $\varphi$-transform techniques; see [10, 11]. From a different aspect, using the Littlewood-Paley $S$-function, Han, Paluszyński and Weiss [16] gave another kind of atomic characterization for the Triebel-Lizorkin space $\dot{F}_{p, q}^{s}\left(\mathbb{R}^{n}\right)$ that is completely analogous to the classical atomic characterization for Hardy spaces, where $s \in \mathbb{R}, p \in(0,1]$ and $q \in[p, \infty)$. (We remark that atomic decompositions of the type considered in [16] were also considered by Frazier and Jawerth [10]; see Theorem 7.4 therein. These non-smooth Frazier-Jawerth atoms are introduced implicitly, using the machinery of the $\phi$-transform, but the decompositions are of the same type as in [16].) Then the question naturally arises whether the boundedness of a sublinear operator in these TriebelLizorkin spaces can be deduced from its uniform boundedness on atoms of Han, Paluszyński and Weiss.

The main purpose of this paper is to answer this question. Indeed, we extend the results of [19] to the Triebel-Lizorkin spaces $\dot{F}_{p, q}^{s}\left(\mathbb{R}^{n}\right)$ with $s \in \mathbb{R}$, $p \in(0,1]$ and $q \in[p, \infty)$ by using the atomic decomposition of these spaces in [16]; see Theorem 2.1, Corollary 2.1 and Theorem 2.2 below. In contrast to the method in [19] which heavily depends on the maximal function characterization of Hardy spaces, here we mainly use the Littlewood-Paley $S$-function characterization of Triebel-Lizorkin spaces. We should mention that some ideas used in this paper come from [19, 3, 16, 25].

The organization of this paper is as follows. In Section 2, we recall some necessary notions including Triebel-Lizorkin spaces and atoms of Han, Paluszyński and Weiss for these spaces, and also state the main results of this paper (Theorems 2.1 and 2.2). In Section 3, we obtain a finite atomic decomposition for a certain dense subspace of the Triebel-Lizorkin space considered, that is, we give the proof of Theorem 2.1. Finally, in Section 4, applying Theorem 2.1, we establish some criterion for boundedness of sublinear operators in Triebel-Lizorkin spaces, that is, we give the proof of Theorem 2.2. We point out that this criterion is useful in the study of boundedness for (sub)linear operators in Triebel-Lizorkin spaces; see, for example, [5, 6, 18].

Throughout this paper, let $\mathbb{N} \equiv\{1,2, \ldots\}, \mathbb{Z}_{+} \equiv \mathbb{N} \cup\{0\}$ and $\mathbb{R}_{+} \equiv$ $[0, \infty)$. Denote by $\sharp E$ the cardinality of any given set $E$. We also denote by $C$ a positive constant independent of the main parameters involved, which may vary at different occurrences. We denote $f \leq C g$ and $f \geq C g$, respectively, by $f \lesssim g$ and $f \gtrsim g$. If $f \lesssim g \lesssim f$, we write $f \sim g$.

2. Main results. To state our main results, we first recall some notation and notions; see, for example, $[10,11,23]$. Denote by $C^{\infty}\left(\mathbb{R}^{n}\right)$ the 
set of infinitely differentiable functions on $\mathbb{R}^{n}$ and $C_{c}^{\infty}\left(\mathbb{R}^{n}\right)$ the set of all $C^{\infty}\left(\mathbb{R}^{n}\right)$ functions with compact support. Let $\mathcal{S}\left(\mathbb{R}^{n}\right)$ be the space of $S$ chwartz functions on $\mathbb{R}^{n}$. Denote by $\mathcal{S}_{\infty}\left(\mathbb{R}^{n}\right)$ the set of functions $\phi \in \mathcal{S}\left(\mathbb{R}^{n}\right)$ such that $\int_{\mathbb{R}^{n}} \phi(x) x^{\gamma} d x=0$ for all multiindices $\gamma \in\left(\mathbb{Z}_{+}\right)^{n}$. Let $\left(\mathcal{S}\left(\mathbb{R}^{n}\right)\right)^{\prime}$ and $\left(\mathcal{S}_{\infty}\left(\mathbb{R}^{n}\right)\right)^{\prime}$ be the dual spaces of $\mathcal{S}\left(\mathbb{R}^{n}\right)$ and $\mathcal{S}_{\infty}\left(\mathbb{R}^{n}\right)$, respectively, and endow them with the weak-* topology. Let $\phi \in \mathcal{S}\left(\mathbb{R}^{n}\right)$ be such that

$$
\operatorname{supp} \widehat{\phi} \subset\left\{x \in \mathbb{R}^{n}: 1 / 2 \leq|x| \leq 2\right\},
$$

and there exists a positive constant $C$ such that for all $3 / 5 \leq|x| \leq 5 / 3$,

$$
|\widehat{\phi}(x)| \geq C ;
$$

here and in what follows, $\widehat{\phi}$ represents the Fourier transform of $\phi$, namely, $\widehat{\phi}(x) \equiv \int_{\mathbb{R}^{n}} \phi(\xi) e^{-i x \cdot \xi} d \xi$. We set $\phi_{j}(x) \equiv 2^{j n} \phi\left(2^{j} x\right)$ for all $x \in \mathbb{R}^{n}$ and $j \in \mathbb{Z}$.

For each cube $Q$ in $\mathbb{R}^{n}$, denote by $c_{Q}$ the center of $Q$ and by $\ell(Q)$ the side length of $Q$. For every $\nu \in \mathbb{Z}$ and $k \in \mathbb{Z}^{n}$, let $Q_{\nu k}$ be the dyadic cube

$$
Q_{\nu k} \equiv\left\{\left(x_{1}, \ldots, x_{n}\right) \in \mathbb{R}^{n}: k_{i} \leq 2^{\nu} x_{i}<k_{i}+1, i=1, \ldots, n\right\} .
$$

Denote by $\mathcal{Q}$ the collection of all dyadic cubes in $\mathbb{R}^{n}$, that is,

$$
\mathcal{Q} \equiv\left\{Q_{\nu k}: \nu \in \mathbb{Z}, k \in \mathbb{Z}^{n}\right\} .
$$

Definition 2.1. Suppose that $\phi \in \mathcal{S}\left(\mathbb{R}^{n}\right)$ satisfies (2.1) and (2.2). For $s \in \mathbb{R}, p \in(0, \infty)$ and $q \in(0, \infty]$, the Triebel-Lizorkin space $\dot{F}_{p, q}^{s}\left(\mathbb{R}^{n}\right)$ is the collection of all $f \in\left(\mathcal{S}_{\infty}\left(\mathbb{R}^{n}\right)\right)^{\prime}$ such that

$$
\|f\|_{\dot{F}_{p, q}^{s}\left(\mathbb{R}^{n}\right)} \equiv\left\|\left(\sum_{\nu \in \mathbb{Z}} 2^{\nu s q}\left|\phi_{\nu} * f\right|^{q}\right)^{1 / q}\right\|_{L^{p}\left(\mathbb{R}^{n}\right)}<\infty,
$$

with the usual modification for $q=\infty$.

REMARK 2.1. In some references (e.g. [10, 11]) in the definition of $\dot{F}_{p, q}^{s}\left(\mathbb{R}^{n}\right),\left(\mathcal{S}_{\infty}\left(\mathbb{R}^{n}\right)\right)^{\prime}$ is replaced by $\left(\mathcal{S}\left(\mathbb{R}^{n}\right)\right)^{\prime} / \mathcal{P}\left(\mathbb{R}^{n}\right)$, where $\mathcal{P}\left(\mathbb{R}^{n}\right)$ denotes the set of all polynomials in $\mathbb{R}^{n}$. Definition 2.1 was given by Triebel [23].

Recall that $\dot{F}_{p, 2}^{0}\left(\mathbb{R}^{n}\right)=L^{p}\left(\mathbb{R}^{n}\right)$ when $p \in(1, \infty)$ and $\dot{F}_{p, 2}^{0}\left(\mathbb{R}^{n}\right)=H^{p}\left(\mathbb{R}^{n}\right)$ when $p \in(0,1)$ (see [23]). It is also known that the definition of TriebelLizorkin space as above is independent of the choice of $\phi$; see, for example $[10,23]$. For $s \in \mathbb{R}$ and $p, q \in(0, \infty)$, the Littlewood-Paley $S$-function (or Lusin function) is used to characterize the Triebel-Lizorkin spaces. Precisely, letting $a$ be some fixed positive constant, and choosing $\phi \in \mathcal{S}\left(\mathbb{R}^{n}\right)$ satisfying (2.1) and (2.2), for $f \in\left(\mathcal{S}_{\infty}\left(\mathbb{R}^{n}\right)\right)^{\prime}$ and $x \in \mathbb{R}^{n}$ we define

$$
\dot{S}_{a, q}^{s}(f)(x) \equiv\left\{\sum_{k \in \mathbb{Z}} \int_{|x-y| \leq a 2^{-k}} 2^{n k+k s q}\left|\left(\phi_{k} * f\right)(y)\right|^{q} d y\right\}^{1 / q} .
$$


Then there exists a positive constant $C$ such that for all $f \in \dot{F}_{p, q}^{s}\left(\mathbb{R}^{n}\right)$,

$$
\frac{1}{C}\|f\|_{\dot{F}_{p, q}^{s}\left(\mathbb{R}^{n}\right)} \leq\left\|\dot{S}_{a, q}^{s}(f)\right\|_{L^{p}\left(\mathbb{R}^{n}\right)} \leq C\|f\|_{\dot{F}_{p, q}^{s}\left(\mathbb{R}^{n}\right)} ;
$$

see, for example, [23] for more details.

To obtain the "atomic" characterization for $\dot{F}_{p, q}^{s}\left(\mathbb{R}^{n}\right)$ where the coefficients, as in the Hardy spaces case, belong to $\ell^{p}$ with $p \in(0,1]$, Han, Paluszyński and Weiss [16] introduce the following atoms.

Definition 2.2. Let $s \in \mathbb{R}, p \in(0,1]$ and $q \in[p, \infty)$. A distribution $a \in\left(\mathcal{S}\left(\mathbb{R}^{n}\right)\right)^{\prime}$ is said to be a $(p, q, s)$-atom of $\dot{F}_{p, q}^{s}\left(\mathbb{R}^{n}\right)$ if

(i) supp $a \subset Q$, where $Q$ is a cube in $\mathbb{R}^{n}$;

(ii) $\|a\|_{\dot{F}_{q, q}^{s}\left(\mathbb{R}^{n}\right)} \leq|Q|^{1 / q-1 / p}$;

(iii) for every $g \in \mathcal{S}\left(\mathbb{R}^{n}\right)$, a polynomial $P$ of degree at most $\mathcal{N} \equiv$ $\max \{\lfloor n(1 / p-1)-s\rfloor,-1\}$ and a smooth cutoff function $\eta_{Q} \in \mathcal{S}\left(\mathbb{R}^{n}\right)$ such that $\eta_{Q} \equiv 1$ on $Q$ and $\eta_{Q} \equiv 0$ outside $2 Q$, we have

$$
\langle a, g\rangle=\left\langle a,(g-P) \eta_{Q}\right\rangle .
$$

Here and in what follows, $2 Q$ denotes the cube centered at $c_{Q}$ and of side length $2 \ell(Q)$, and $P$ disappears if $\mathcal{N}=-1$. In the above formula, $\langle f, \phi\rangle$ denotes the natural pairing of $f \in\left(\mathcal{S}\left(\mathbb{R}^{n}\right)\right)^{\prime}$ and $\phi \in \mathcal{S}\left(\mathbb{R}^{n}\right)$, and $\langle f, \phi\rangle \equiv$ $\int_{\mathbb{R}^{n}} f(x) \phi(x) d x$ when $f$ is a function.

Observe that if a $(p, q, s)$-atom of $\dot{F}_{p, q}^{s}\left(\mathbb{R}^{n}\right)$ is locally integrable, then condition (iii) in Definition 2.2 is again the usual cancellation condition; see [16]. Moreover, the $(p, 2,0)$-atoms of $\dot{F}_{p, q}^{s}\left(\mathbb{R}^{n}\right)$ are just the classical atoms of Hardy spaces $H^{p}\left(\mathbb{R}^{n}\right)$ by recalling that $\dot{F}_{2,2}^{0}\left(\mathbb{R}^{n}\right)=L^{2}\left(\mathbb{R}^{n}\right)$ (see [23]).

Han, Paluszyński and Weiss [16, Theorem 1] established the following "atomic" decomposition for $\dot{F}_{p, q}^{s}\left(\mathbb{R}^{n}\right)$ when $s \in \mathbb{R}, p \in(0,1]$ and $q \in[p, \infty)$.

Lemma 2.1. Let $s \in \mathbb{R}, p \in(0,1]$ and $q \in[p, \infty)$. Then $f \in \dot{F}_{p, q}^{s}\left(\mathbb{R}^{n}\right)$ if and only if there exist $\left\{\lambda_{k}\right\}_{k \in \mathbb{Z}} \subset \mathbb{C}$ and $(p, q, s)$-atoms $\left\{a_{k}\right\}_{k \in \mathbb{N}}$ such that $\sum_{k \in \mathbb{N}}\left|\lambda_{k}\right|^{p}<\infty$ and $f=\sum_{k \in \mathbb{N}} \lambda_{k} a_{k}$ in $\left(\mathcal{S}_{\infty}\left(\mathbb{R}^{n}\right)\right)^{\prime}$. Moreover, there exists a positive constant $C$ such that for all $f \in \dot{F}_{p, q}^{s}\left(\mathbb{R}^{n}\right)$,

$$
\frac{1}{C}\|f\|_{\dot{F}_{p, q}^{s}\left(\mathbb{R}^{n}\right)} \leq \inf \left\{\left(\sum_{k \in \mathbb{N}}\left|\lambda_{k}\right|^{p}\right)^{1 / p}\right\} \leq C\|f\|_{\dot{F}_{p, q}^{s}\left(\mathbb{R}^{n}\right)},
$$

where the infimum is taken over all the decompositions of $f$ as above.

Let $s \in \mathbb{R}, p \in(0,1]$ and $q \in[p, \infty)$. Denote by $\dot{F}_{p, q}^{s, \text { fin }}\left(\mathbb{R}^{n}\right)$ the vector space of all finite linear combinations of infinitely differentiable $(p, q, s)$ - 
atoms of $\dot{F}_{p, q}^{s}\left(\mathbb{R}^{n}\right)$ endowed with the quasi-norm

$$
\begin{aligned}
& \|f\|_{\dot{F}_{p, q}^{s, \text { fin }}\left(\mathbb{R}^{n}\right)} \\
& \quad \equiv \inf \left\{\left(\sum_{j=1}^{N}\left|\lambda_{j}\right|^{p}\right)^{1 / p}: f=\sum_{j=1}^{N} \lambda_{j} a_{j}, N \in \mathbb{N},\left\{\lambda_{j}\right\}_{j=1}^{N} \subset \mathbb{C},\right. \\
& \left.\left\{a_{j}\right\}_{j=1}^{N} \text { are infinitely differentiable }(p, q, s) \text {-atoms of } \dot{F}_{p, q}^{s}\left(\mathbb{R}^{n}\right)\right\} .
\end{aligned}
$$

For $s \in \mathbb{R}, p \in(0,1]$ and $q \in[p, \infty)$, it follows from Lemma 4.1 below that $\dot{F}_{p, q}^{s, f i n}\left(\mathbb{R}^{n}\right)$ is a dense subset of $\dot{F}_{p, q}^{s}\left(\mathbb{R}^{n}\right)$. Moreover, by Lemma 2.1, there exists a positive constant $C$ such that for all $f \in \dot{F}_{p, q}^{s, f i n}\left(\mathbb{R}^{n}\right)$,

$$
\|f\|_{\dot{F}_{p, q}^{s}\left(\mathbb{R}^{n}\right)} \leq C\|f\|_{\dot{F}_{p, q}^{s, f i n}\left(\mathbb{R}^{n}\right)} .
$$

The converse inequality for all $f \in \dot{F}_{p, q}^{s, \text { fin }}\left(\mathbb{R}^{n}\right)$ is established in the following theorem.

TheOREM 2.1. Let $s \in \mathbb{R}, p \in(0,1]$ and $q \in[p, \infty)$. Then for any given $f \in \dot{F}_{p, q}^{s, \mathrm{fin}}\left(\mathbb{R}^{n}\right)$, there exist some $N \in \mathbb{N}$, a sequence $\left\{\lambda_{k}\right\}_{k=1}^{N} \subset \mathbb{C}$ and infinitely differentiable $(p, q, s)$-atoms $\left\{a_{k}\right\}_{k=1}^{N}$ of $\dot{F}_{p, q}^{s}\left(\mathbb{R}^{n}\right)$ such that $f=$ $\sum_{k=1}^{N} \lambda_{k} a_{k}$ pointwise, and

$$
\left(\sum_{k=1}^{N}\left|\lambda_{k}\right|^{p}\right)^{1 / p} \leq C\|f\|_{\dot{F}_{p, q}^{s}\left(\mathbb{R}^{n}\right)},
$$

where $C$ is a positive constant independent of $f$.

Here we describe some ideas used in the proof of Theorem 2.1 in Section 3. Using the Calderón reproducing formula (see Lemma 3.2 below), we write $f$ as a sum over all dyadic cubes in $\mathbb{R}^{n}$ (see (3.3)), which essentially gives the atomic decomposition of $f$ (see [16] or the property (iii) in the proof of Theorem 2.1). To obtain a finite atomic decomposition of $f$, we set

$$
Q\left(0,2^{N}\right) \equiv\left\{\left(x_{1}, \ldots, x_{n}\right) \in \mathbb{R}^{n}:-1 \leq 2^{-N} x_{i}<1, i=1, \ldots, n\right\},
$$

and then we carefully classify all dyadic cubes in $\mathbb{R}^{n}$ (see (3.4)). Based on this subtle classification, we write $f=f_{N}+b_{N}$ as in (3.7), where $f_{N}$ is a linear combination of finitely many $(p, q, s)$-atoms of $\dot{F}_{p, q}^{s}\left(\mathbb{R}^{n}\right)$ and the support of each atom lies in a multiple of some dyadic cube $Q$ with $Q \subset Q\left(0,2^{N}\right)$ and $\ell(Q) \geq 2^{-N}$. So our task is then to show that $b_{N}$ is an arbitrarily small multiple of a certain $(p, q, s)$-atom of $\dot{F}_{p, q}^{s}\left(\mathbb{R}^{n}\right)$ for large $N$. From this, we can deduce the desired conclusion of Theorem 2.1; see Section 3 for the details.

The following conclusion is an easy corollary of Theorem 2.1 and (2.6). We omit the details. 
Corollary 2.1. Let $s \in \mathbb{R}, p \in(0,1]$ and $q \in[p, \infty)$. Then there exists a positive constant $C$ such that for all $f \in \dot{F}_{p, q}^{s, \text { fin }}\left(\mathbb{R}^{n}\right)$,

$$
\frac{1}{C}\|f\|_{\dot{F}_{p, q}^{s}\left(\mathbb{R}^{n}\right)} \leq\|f\|_{\dot{F}_{p, q}^{s, \text { in }}\left(\mathbb{R}^{n}\right)} \leq C\|f\|_{\dot{F}_{p, q}^{s}\left(\mathbb{R}^{n}\right)} .
$$

As an application of Theorem 2.1 and Corollary 2.1, we obtain a boundedness criterion for sublinear operators from $\dot{F}_{p, q}^{s}\left(\mathbb{R}^{n}\right)$ to quasi-Banach spaces. To state it, we need the following notions; see, for example, [26].

Definition 2.3. (i) A quasi-Banach space $\mathcal{B}$ is a vector space endowed with a quasi-norm $\|\cdot\|_{\mathcal{B}}$ which is non-negative, non-degenerate (i.e., $\|f\|_{\mathcal{B}}=0$ if and only if $f=0$ ), homogeneous, and obeys the quasi-triangle inequality, i.e., there exists a constant $K \geq 1$ such that $\|f+g\|_{\mathcal{B}} \leq K\left(\|f\|_{\mathcal{B}}+\|g\|_{\mathcal{B}}\right)$ for all $f, g \in \mathcal{B}$.

(ii) Let $r \in(0,1]$. A quasi-Banach space $\mathcal{B}_{r}$ with the quasi norm $\|\cdot\|_{\mathcal{B}_{r}}$ is said to be an $r$-quasi-Banach space if

$$
\|f+g\|_{\mathcal{B}_{r}}^{r} \leq\|f\|_{\mathcal{B}_{r}}^{r}+\|g\|_{\mathcal{B}_{r}}^{r} \quad \text { for all } f, g \in \mathcal{B}_{r} .
$$

(iii) For any given $r$-quasi-Banach space $\mathcal{B}_{r}$ with $r \in(0,1]$ and a linear space $\mathcal{Y}$, an operator $T$ from $\mathcal{Y}$ to $\mathcal{B}_{r}$ is called $\mathcal{B}_{r}$-sublinear if for all $f, g \in \mathcal{Y}$ and $\lambda, \nu \in \mathbb{C}$,

$$
\|T(\lambda f+\nu g)\|_{\mathcal{B}_{r}} \leq\left(|\lambda|^{r}\|T(f)\|_{\mathcal{B}_{r}}^{r}+|\nu|^{r}\|T(g)\|_{\mathcal{B}_{r}}^{r}\right)^{1 / r}
$$

and

$$
\|T(f)-T(g)\|_{\mathcal{B}_{r}} \leq\|T(f-g)\|_{\mathcal{B}_{r}} .
$$

Theorem 2.2. Let $s \in \mathbb{R}, p \in(0,1], q \in[p, \infty), r \in[p, 1]$ and $\mathcal{B}_{r}$ be an $r$-quasi-Banach space. If $T: \dot{F}_{p, q}^{s, f i n}\left(\mathbb{R}^{n}\right) \rightarrow \mathcal{B}_{r}$ is a $\mathcal{B}_{r}$-sublinear operator such that

$$
\begin{array}{r}
\sup \left\{\|T a\|_{\mathcal{B}_{r}}:\right. \text { a is an infinitely differentiable } \\
\left.\qquad(p, q, s) \text {-atom of } \dot{F}_{p, q}^{s}\left(\mathbb{R}^{n}\right)\right\}<\infty,
\end{array}
$$

then $T$ uniquely extends to a bounded $\mathcal{B}_{r}$-sublinear operator from $\dot{F}_{p, q}^{s}\left(\mathbb{R}^{n}\right)$ to $\mathcal{B}_{r}$.

REMARK 2.2. (a) Let $p, q, s$ and $r$ be as in Theorem 2.2. If $T$ is a bounded $\mathcal{B}_{r}$-sublinear operator from $\dot{F}_{p, q}^{s}\left(\mathbb{R}^{n}\right)$ to $\mathcal{B}_{r}$, by Lemma 2.1 we know that $T$ satisfies (2.9). Thus (2.9) is also necessary for the boundedness of $T$ from $\dot{F}_{p, q}^{s}\left(\mathbb{R}^{n}\right)$ to $\mathcal{B}_{r}$.

(b) Any Banach space is a 1-quasi-Banach space, and the Triebel-Lizorkin spaces $\dot{F}_{p, q}^{s}\left(\mathbb{R}^{n}\right)$ with $s \in \mathbb{R}, p \in(0,1]$ and $q \in[p, \infty)$ are typical $p$-quasiBanach spaces.

(c) Obviously, if $T$ is linear, then $T$ is $\mathcal{B}_{r}$-sublinear. Moreover, if $\mathcal{B}_{r}$ is a space of functions and $T$ is sublinear in the classical sense, and $T(f) \geq 0$ for all $f \in \mathcal{Y}$, then $T$ is also $\mathcal{B}_{r}$-sublinear. 
(d) According to the Aoki-Rolewicz theorem (see [1] or [22]), any quasiBanach space is, essentially, an $r$-quasi-Banach space, where

$$
r=1 / \log _{2}(2 K)
$$

and $K$ is as in Definition 2.3(i). Thus, Theorem 2.2 eventually holds for all quasi-Banach spaces satisfying $K \in\left[1,2^{1 / p-1}\right]$.

3. Proof of Theorem 2.1. The main purpose of this section is to prove Theorem 2.1. The following lemma is a variant of [11, Lemma (5.12)], which is used to obtain the Calderón reproducing formula. A detailed proof is included here for the reader's convenience; see also [9, Theorem 2.6] and [11, Lemma (1.1)].

Lemma 3.1. For any given $L \in \mathbb{Z}_{+}$, there exist real-valued radial functions $\psi \in C_{c}^{\infty}\left(\mathbb{R}^{n}\right)$ and $\varphi \in \mathcal{S}_{\infty}\left(\mathbb{R}^{n}\right)$ such that

(i) $\operatorname{supp} \psi \subset B(0,1) \equiv\left\{x \in \mathbb{R}^{n}:|x|<1\right\}$;

(ii) $\int_{\mathbb{R}^{n}} \psi(x) x^{\gamma} d x=0$ for all $\gamma \in\left(\mathbb{Z}_{+}\right)^{n}$ and $|\gamma| \leq L$;

(iii) $\widehat{\psi} \geq 0$ and there exists a positive constant $C$ such that $\widehat{\psi}(\xi) \geq C$ for all $1 / 2 \leq|\xi| \leq 2$;

(iv) $\widehat{\varphi} \geq 0, \operatorname{supp} \widehat{\varphi} \subset\left\{\xi \in \mathbb{R}^{n}: 1 / 2 \leq|\xi| \leq 2\right\}$ and there exists a positive constant $C$ such that $\widehat{\varphi}(\xi) \geq C$ for all $3 / 5 \leq|\xi| \leq 5 / 3$;

(v) $\sum_{j \in \mathbb{Z}} \widehat{\psi}\left(2^{-j} \xi\right) \widehat{\varphi}\left(2^{-j} \xi\right)=1$ for all $\xi \in \mathbb{R}^{n} \backslash\{0\}$.

Proof. Choose $N \in \mathbb{N}$ satisfying $N \geq\lfloor L / 2\rfloor+1$. Let $\theta \in C_{c}^{\infty}\left(\mathbb{R}^{n}\right)$ be a real-valued radial function supported on $\left\{x \in \mathbb{R}^{n}:|x|<1 / 2\right\}$ and $\widehat{\theta}(0)=1$. Notice that $\theta$ being real-valued and radial implies that $\widehat{\theta}$ is also real-valued and radial. So there exists $\varepsilon>0$ such that $\widehat{\theta}(x)>1 / 2$ for all $|x| \leq 2 \varepsilon$. Set $h \equiv(-\Delta)^{N} \theta_{\varepsilon}$, where $\Delta$ is the Laplace operator and $\theta_{\varepsilon}(x) \equiv \varepsilon^{-n} \theta(x / \varepsilon)$ for all $x \in \mathbb{R}^{n}$. Integration by parts shows that $h$ satisfies (ii). For all $x \in \mathbb{R}^{n}$ with $1 / 2 \leq|x| \leq 2$, we have $\widehat{h}(x)=|x|^{2 N} \widehat{\theta}(\varepsilon x) \geq 8^{-N}$. Set $\psi \equiv h * h$. Then it is easy to verify that $\psi$ is a real-valued radial function satisfying (i), (ii), $\widehat{\psi} \geq 0$ and

$$
\widehat{\psi}(x) \geq 64^{-N} \quad \text { for all } 1 / 2 \leq|x| \leq 2 .
$$

Now we select a non-negative radial function $\eta$ with $\operatorname{supp} \eta \subset\left\{x \in \mathbb{R}^{n}\right.$ : $1 / 2 \leq|x| \leq 2\}$ and $\eta(x) \geq 1 / 2$ for all $3 / 5 \leq|x| \leq 5 / 3$. Then we set $g(\xi) \equiv$ $\sum_{k \in \mathbb{Z}} \eta\left(2^{k} \xi\right) \widehat{\psi}\left(2^{k} \xi\right)$ for all $\xi \in \mathbb{R}^{n}$. This combined with (3.1) implies that there exists a positive constant $C$ depending on $L$ such that $C^{-1} \leq g(\xi) \leq C$ for all $\xi \in \mathbb{R}^{n} \backslash\{0\}$. Let $\varphi$ be given by $\widehat{\varphi}(\xi) \equiv \eta(\xi) / g(\xi)$ for all $\xi \in \mathbb{R}^{n}$. Then it is easy to deduce that $\varphi$ satisfies (iv) and (v). This finishes the proof of Lemma 3.1. 
By Lemma 3.1 and an argument as in the proof of $[11$, p. 122 , Theorem 3], we obtain the following Calderón reproducing formula, which is the fundamental tool used to obtain the atomic decompositions of $\dot{F}_{p, q}^{s}\left(\mathbb{R}^{n}\right)$. The proof of Lemma 3.2 is omitted since it is similar to that of $[11$, p. 120, Theorem 1] and [11, p. 122, Theorem 3].

Lemma 3.2. Let $\varphi \in \mathcal{S}_{\infty}\left(\mathbb{R}^{n}\right)$ and $\psi \in C_{c}^{\infty}\left(\mathbb{R}^{n}\right)$ be as in Lemma 3.1. Then

(i) if $f \in \mathcal{S}\left(\mathbb{R}^{n}\right)$, then for all $x \in \mathbb{R}^{n}$,

$$
f(x)=\sum_{k \in \mathbb{Z}}\left(\psi_{k} * \varphi_{k} * f\right)(x) ;
$$

(ii) if $f \in \mathcal{S}_{\infty}\left(\mathbb{R}^{n}\right)$, then (3.2) holds in $\mathcal{S}_{\infty}\left(\mathbb{R}^{n}\right)$; and if $f \in\left(\mathcal{S}_{\infty}\left(\mathbb{R}^{n}\right)\right)^{\prime}$, then (3.2) holds in $\left(\mathcal{S}_{\infty}\left(\mathbb{R}^{n}\right)\right)^{\prime}$.

For any $L \in \mathbb{Z}_{+}$, denote by $\mathcal{S}_{L}\left(\mathbb{R}^{n}\right)$ the space of all functions in $\mathcal{S}\left(\mathbb{R}^{n}\right)$ with vanishing moments up to order $L$, i.e.,

$$
\mathcal{S}_{L}\left(\mathbb{R}^{n}\right) \equiv\left\{\varphi \in \mathcal{S}\left(\mathbb{R}^{n}\right): \int_{\mathbb{R}^{n}} \varphi(x) x^{\gamma} d x=0 \text { for all }|\gamma| \leq L\right\} .
$$

When $L=-1$, we set $\mathcal{S}_{-1}\left(\mathbb{R}^{n}\right) \equiv \mathcal{S}\left(\mathbb{R}^{n}\right)$. Recall that for any function $\phi$, we set $\phi_{k}(x) \equiv 2^{k n} \phi\left(2^{k} x\right)$ for all $x \in \mathbb{R}^{n}$ and $k \in \mathbb{Z}$. The following technical lemma plays a crucial role in the proof of Theorem 2.1.

LEMMA 3.3.

(i) Let $g \in \mathcal{S}\left(\mathbb{R}^{n}\right)$ and $\psi \in \mathcal{S}_{L}\left(\mathbb{R}^{n}\right)$ with $L \in \mathbb{Z}_{+}$. Then for any given $M_{1}, L_{1} \in \mathbb{Z}_{+}$with $L_{1} \leq L$, there exists a positive constant $C$ such that for all $k \in \mathbb{Z}_{+}$and $x \in \mathbb{R}^{n}$,

$$
\left|\left(\psi_{k} * g\right)(x)\right| \leq C 2^{-k L_{1}}(1+|x|)^{-M_{1}} .
$$

(ii) Let $g \in \mathcal{S}_{L}\left(\mathbb{R}^{n}\right)$ with $L \in \mathbb{Z}_{+} \cup\{-1\}$ and $\psi \in \mathcal{S}_{0}\left(\mathbb{R}^{n}\right)$. Then for any given $M_{2} \in \mathbb{Z}_{+}, L_{2} \in \mathbb{Z}_{+} \cup\{-1\}$ with $L_{2} \leq L$, there exists a positive constant $C$ such that for all $k \leq 0$ and $x \in \mathbb{R}^{n}$,

$$
\left|\left(\psi_{k} * g\right)(x)\right| \leq C 2^{k\left(n+L_{2}+1\right)}\left(1+2^{k}|x|\right)^{-M_{2}} .
$$

Proof. By a procedure as in [11, p. 121, Lemma 2], we obtain (i). Property (ii) is essentially given in [11, p. 122, Lemma 4].

Let $\phi \in \mathcal{S}_{\infty}\left(\mathbb{R}^{n}\right)$ and $\psi \in \mathcal{S}_{L}\left(\mathbb{R}^{n}\right)$ with $L \in \mathbb{Z}_{+}$. Notice that for all $\nu$, $j \in \mathbb{Z}$ and $z \in \mathbb{R}^{n}, \phi_{\nu} * \psi_{j}(z)=2^{\nu n}\left(\psi_{j-\nu} * \phi\right)\left(2^{\nu} z\right)$. From this and Lemma 3.3 , we directly deduce the following conclusion. 
Corollary 3.1. Let $\phi \in \mathcal{S}_{\infty}\left(\mathbb{R}^{n}\right)$ and $\psi \in \mathcal{S}_{L}\left(\mathbb{R}^{n}\right)$ with $L \in \mathbb{Z}_{+}$. Then

(i) for any given $M_{3}, L_{3} \in \mathbb{Z}_{+}$with $L_{3} \leq L$, there exists a positive constant $C$ such that for all $\nu \leq j$ and $z \in \mathbb{R}^{n}$,

$$
\left|\left(\phi_{\nu} * \psi_{j}\right)(z)\right| \leq C 2^{\nu n} 2^{-(j-\nu) L_{3}}\left(1+2^{\nu}|z|\right)^{-M_{3}} ;
$$

(ii) for any given $M_{4}, L_{4} \in \mathbb{Z}_{+}$, there exists a positive constant $C$ such that for all $\nu>j$ and $z \in \mathbb{R}^{n}$,

$$
\left|\left(\phi_{\nu} * \psi_{j}\right)(z)\right| \leq C 2^{\nu n} 2^{-(\nu-j)\left(n+L_{4}+1\right)}\left(1+2^{j}|z|\right)^{-M_{4}} .
$$

Now we turn to the proof of Theorem 2.1.

Proof of Theorem 2.1. Let $f \in \dot{F}_{p, q}^{s, \text { fin }}\left(\mathbb{R}^{n}\right)$ and $f \not \equiv 0$. Let $a$ be some fixed positive number and $\dot{S}_{a, q}^{s}(f)$ as in (2.3). By (2.6) and (2.4), $\dot{S}_{a, q}^{s}(f) \in$ $L^{p}\left(\mathbb{R}^{n}\right)$. Using the idea in [4], for any $k \in \mathbb{Z}$, we set

$$
\Omega_{k} \equiv\left\{x \in \mathbb{R}^{n}: \dot{S}_{a, q}^{s}(f)(x)>2^{k}\right\}
$$

and

$$
\mathcal{Q}_{k} \equiv\left\{Q \in \mathcal{Q}:\left|Q \cap \Omega_{k}\right|>|Q| / 2,\left|Q \cap \Omega_{k+1}\right| \leq|Q| / 2\right\} .
$$

It is easy to see that for each dyadic cube $Q \in \mathcal{Q}$, there exists a unique $k \in \mathbb{Z}$ such that $Q \in \mathcal{Q}_{k}$. A dyadic cube $Q \in \mathcal{Q}_{k}$ is said to be maximal if for any dyadic cube $Q^{\prime} \in \mathcal{Q}_{k}$, either $Q^{\prime} \subset Q$ or $Q^{\prime} \cap Q=\emptyset$. For each $k \in \mathbb{Z}$, denote by $\left\{Q_{k}^{i}\right\}_{i \in I_{k}}$ the collection of all maximal dyadic cubes in $\mathcal{Q}_{k}$, where the index set $I_{k}$ may be empty. Then

$$
\mathcal{Q}=\bigcup_{k \in \mathbb{Z}} \mathcal{Q}_{k}=\bigcup_{k \in \mathbb{Z}} \bigcup_{i \in I_{k}}\left\{Q \in \mathcal{Q}_{k}: Q \subset Q_{k}^{i}\right\} .
$$

Let $\psi$ and $\varphi$ be as in Lemma 3.2, where $\varphi \in \mathcal{S}_{\infty}\left(\mathbb{R}^{n}\right)$ and $\psi \in \mathcal{S}_{L_{0}}\left(\mathbb{R}^{n}\right)$ with $L_{0} \in \mathbb{N}$. We may as well assume that $L_{0}>\mathcal{N}$ is a very large natural number. Set $\psi_{Q} \equiv \psi_{\ell}$ and $\varphi_{Q} \equiv \varphi_{\ell}$ whenever $\ell(Q)=2^{-\ell}$. Using Lemma $3.2(\mathrm{i})$, for all $x \in \mathbb{R}^{n}$, we have

$$
\begin{aligned}
f(x) & =\sum_{k \in \mathbb{Z}} \sum_{\left\{Q \in \mathcal{Q}: \ell(Q)=2^{-k}\right\}} \int_{Q} \psi_{k}(x-y)\left(\varphi_{k} * f\right)(y) d y \\
& =\sum_{Q \in \mathcal{Q} Q} \int_{Q} \psi_{Q}(x-y)\left(\varphi_{Q} * f\right)(y) d y \\
& =\sum_{k \in \mathbb{Z}} \sum_{i \in I_{k}}\left\{\sum_{Q \subset Q_{k}^{i}, Q \in \mathcal{Q}_{k}} \int_{Q} \psi_{Q}(x-y)\left(\varphi_{Q} * f\right)(y) d y\right\} .
\end{aligned}
$$

When $q \in[1, \infty)$, we set

$$
\lambda_{k, i} \equiv C\left|Q_{k}^{i}\right|^{1 / p-1 / q}\left\{\sum_{Q \subset Q_{k}^{i}, Q \in \mathcal{Q}_{k}} \int_{Q} \ell(Q)^{-s q}\left|\left(\varphi_{Q} * f\right)(y)\right|^{q} d y\right\}^{1 / q} ;
$$


and when $q \in[p, 1)$, we set

$$
\lambda_{k, i} \equiv C\left|Q_{k}^{i}\right|^{1 / p-1 / q}\left\{\sum_{Q \subset Q_{k}^{i}, Q \in \mathcal{Q}_{k}}|Q| \sup _{y \in Q}\left(\ell(Q)^{-s}\left|\left(\varphi_{Q} * f\right)(y)\right|\right)^{q}\right\}^{1 / q},
$$

where $C$ is some positive constant independent of $k, i$ and $f$. Set

$$
a_{k, i}(x) \equiv \frac{1}{\lambda_{k, i}}\left\{\sum_{Q \subset Q_{k}^{i}, Q \in \mathcal{Q}_{k}} \int_{Q} \psi_{Q}(x-y)\left(\varphi_{Q} * f\right)(y) d y\right\} .
$$

Using (3.3), (2.4) and following closely the proof of [16, Theorem 1], we obtain:

(i) each $a_{k, i}$ is a $(p, q, s)$-atom of $\dot{F}_{p, q}^{s}\left(\mathbb{R}^{n}\right)$ supported on $(\sqrt{n} / 2+1) Q_{k}^{i}$;

(ii) there exists a positive constant $C$ such that $\sum_{k \in \mathbb{Z}} \sum_{i \in I_{k}}\left|\lambda_{k, i}\right|^{p} \leq$ $C\|f\|_{\dot{F}_{p, q}^{s}\left(\mathbb{R}^{n}\right)}^{p}$

(iii) for all $x \in \mathbb{R}^{n}, f(x)=\sum_{k \in \mathbb{Z}} \sum_{i \in I_{k}} \lambda_{k, i} a_{k, i}(x)$.

To obtain a finite atomic decomposition of $f$, we need a new classification of all dyadic cubes in $\mathbb{R}^{n}$. For every integer $N \in \mathbb{N}$, set

$$
Q\left(0,2^{N}\right) \equiv\left\{\left(x_{1}, \ldots, x_{n}\right) \in \mathbb{R}^{n}:-1 \leq 2^{-N} x_{i}<1, i=1, \ldots, n\right\},
$$

and

$$
W_{1}^{N} \equiv\left\{Q \in \mathcal{Q}: Q \subset Q\left(0,2^{N}\right), \ell(Q) \geq 2^{-N}\right\}, \quad W_{2}^{N} \equiv \mathcal{Q} \backslash W_{1}^{N} .
$$

Notice that $W_{1}^{N}$ has finitely many elements. For each $Q \in W_{1}^{N}$, there exist unique $k \in \mathbb{Z}$ and $i \in I_{k}$ such that $Q \subset Q_{k}^{i}$. Denote by $J_{N}$ the collection of all such $(k, i)$. Obviously, $\sharp J_{N} \leq \sharp W_{1}^{N}$ and thus $J_{N}$ is finite. For every $Q_{k}^{i}$ with $(k, i) \in J_{N}$, let

$$
\widetilde{\mathcal{Q}_{k}^{i}} \equiv\left\{Q \in \mathcal{Q}: Q \subset Q_{k}^{i}, Q \in \mathcal{Q}_{k}, Q \subset Q\left(0,2^{N}\right), \ell(Q) \geq 2^{-N}\right\} .
$$

Notice that

$$
W_{1}^{N}=\bigcup_{(k, i) \in J_{N}}\left\{Q \in \mathcal{Q}: Q \in \widetilde{\mathcal{Q}_{k}^{i}}\right\}
$$

For every $(k, i) \in J_{N}$, we set

$$
\widetilde{a}_{k, i}(x) \equiv \frac{1}{\lambda_{k, i}} \sum_{Q \in \widetilde{\mathcal{Q}_{k}^{i}}} \int_{Q} \psi_{Q}(x-y)\left(\varphi_{Q} * f\right)(y) d y .
$$

In a way similar to the proof of $a_{k, i}$ being a $(p, q, s)$-atom of $\dot{F}_{p, q}^{s}\left(\mathbb{R}^{n}\right)$ (see [16, Theorem 1]), we can easily verify that each $\widetilde{a}_{k, i}$ is still a $(p, q, s)$-atom of $\dot{F}_{p, q}^{s}\left(\mathbb{R}^{n}\right)$. For any $(k, i) \in J_{N}$, as $f \in C_{c}^{\infty}\left(\mathbb{R}^{n}\right), \psi \in \mathcal{S}_{L_{0}}\left(\mathbb{R}^{n}\right), \varphi \in \mathcal{S}_{\infty}\left(\mathbb{R}^{n}\right)$ 
and $\sharp \widetilde{\mathcal{Q}_{k}^{i}} \leq \sharp W_{1}^{N}<\infty$, we deduce that $\widetilde{a}_{k, i} \in C_{c}^{\infty}\left(\mathbb{R}^{n}\right)$. Set

$$
f_{N} \equiv \sum_{(k, i) \in J_{N}} \lambda_{k, i} \widetilde{a}_{k, i} \quad \text { and } \quad b_{N} \equiv f-f_{N} \text {. }
$$

Then $f_{N}$ is a linear combination of finitely many $(p, q, s)$-atoms of $\dot{F}_{p, q}^{s}\left(\mathbb{R}^{n}\right)$, and the property (ii) above shows that the $\ell^{p}$-norm of its coefficients is bounded by a multiple of $\|f\|_{\dot{F}_{p, q}^{s}\left(\mathbb{R}^{n}\right)}$. The definition of $f_{N}$ together with (3.5) and (3.6) implies that

$$
f_{N}(x)=\sum_{Q \in W_{1}^{N}} \int_{Q} \psi_{Q}(x-y)\left(\varphi_{Q} * f\right)(y) d y .
$$

This combined with Lemma 3.1(i) and the definition of $W_{1}^{N}$ shows that supp $f_{N} \subset B\left(0, C_{n} 2^{N}\right)$, where $C_{n}$ is a positive constant depending only on the dimension $n$. The assumption $f \in \dot{F}_{p, q}^{s, \text { fin }}\left(\mathbb{R}^{n}\right)$ implies that there exists $R>0$ such that supp $f \subset B(0, R)$. From this and $b_{N}=f-f_{N}$, it follows that there exists some $N_{0} \in \mathbb{N}$ large enough such that $\operatorname{supp} b_{N} \subset B\left(0, C_{n} 2^{N}\right)$ when $N>N_{0}$. Notice that $b_{N} \in C_{c}^{\infty}\left(\mathbb{R}^{n}\right)$ and $b_{N}$ has vanishing moments up to order $\mathcal{N}$ since $f$ and $f_{N}$ do.

We further claim that there exist constants $\sigma \in(0,1)$ and $\widetilde{C}>0$ such that for all $N>N_{0}$,

$$
\left\|b_{N}\right\|_{\dot{F}_{q, q}^{s}} \leq \widetilde{C} 2^{-N \sigma}\left|B\left(0, C_{n} 2^{N}\right)\right|^{1 / q-1 / p} .
$$

Assume that (3.9) holds for the moment. Set $a_{N} \equiv \widetilde{C}^{-1} 2^{N \sigma} b_{N}$. Then $a_{N}$ is a $(p, q, s)$-atom of $\dot{F}_{p, q}^{s}\left(\mathbb{R}^{n}\right)$ for $N$ large enough. Therefore, for large $N$, we have

$$
f=f_{N}+b_{N}=\sum_{(k, i) \in J_{N}} \lambda_{k, i} \widetilde{a}_{k, i}+\widetilde{C} 2^{-N \sigma} a_{N}
$$

which is a linear combination of finitely many $(p, q, s)$-atoms of $\dot{F}_{p, q}^{s}\left(\mathbb{R}^{n}\right)$, each atom belongs to $C_{c}^{\infty}\left(\mathbb{R}^{n}\right)$ and the $\ell^{p}$-norm of its coefficients is bounded by a multiple of $\|f\|_{\dot{F}_{p, q}^{s}\left(\mathbb{R}^{n}\right)}$. This implies the desired result of Theorem 2.1.

To complete the proof of Theorem 2.1, we still need to verify (3.9). By (3.3), (3.4), (3.7) and (3.8), we obtain

$$
b_{N}(x)=\sum_{Q \in W_{2}^{N}} \int_{Q} \psi_{Q}(x-y)\left(\varphi_{Q} * f\right)(y) d y .
$$

Notice that

$$
\begin{aligned}
W_{2}^{N}= & \left\{Q \in \mathcal{Q}: Q \cap Q\left(0,2^{N}\right)=\emptyset, 2^{-N} \leq \ell(Q) \leq 2^{N}\right\} \\
& \cup\left\{Q \in \mathcal{Q}: \ell(Q)<2^{-N} \text { or } \ell(Q)>2^{N}\right\} .
\end{aligned}
$$


From this and (3.11), it follows that

$$
\begin{aligned}
b_{N}(x)= & \left\{\sum_{|j| \leq N} \sum_{\substack{\ell(Q)=2^{-j} \\
Q \cap Q\left(0,2^{N}\right)=\emptyset}}+\sum_{|j|>N} \sum_{\ell(Q)=2^{-j}}\right\} \\
& \times \int_{Q} \psi_{Q}(x-y)\left(\varphi_{Q} * f\right)(y) d y \\
= & \sum_{|j| \leq N \mathbb{R}^{n} \backslash Q\left(0,2^{N}\right)} \psi_{j}(x-y)\left(\varphi_{j} * f\right)(y) d y \\
& +\sum_{|j|>N} \int_{\mathbb{R}^{n}} \psi_{j}(x-y)\left(\varphi_{j} * f\right)(y) d y .
\end{aligned}
$$

Let $\phi$ be as in Definition 2.1. Observe that by Lemma 3.2(ii), we see that (3.3), and therefore, (3.11) hold in $\left(\mathcal{S}_{\infty}\left(\mathbb{R}^{n}\right)\right)^{\prime}$. From this, Definition 2.1 and (3.12), it follows that

$$
\begin{aligned}
\left\|b_{N}\right\|_{\dot{F}_{q, q}^{s}\left(\mathbb{R}^{n}\right)} & =\left\{\sum_{\nu \in \mathbb{Z}} 2^{\nu s q} \int_{\mathbb{R}^{n}}\left|\left(\phi_{\nu} * b_{N}\right)(x)\right|^{q} d x\right\}^{1 / q} \\
\lesssim & \left\{\sum_{\nu \in \mathbb{Z}} 2^{\nu s q} \int_{\mathbb{R}^{n}}\left|\sum_{|j| \leq N} \int_{\mathbb{R}^{n} \backslash Q\left(0,2^{N}\right)}\left(\phi_{\nu} * \psi_{j}\right)(x-y)\left(\varphi_{j} * f\right)(y) d y\right|^{q} d x\right\}^{1 / q} \\
& +\left\{\sum_{\nu \in \mathbb{Z}} 2^{\nu s q} \int_{\mathbb{R}^{n}}\left|\sum_{|j|>N}\left(\phi_{\nu} * \psi_{j} * \varphi_{j} * f\right)(x)\right|^{q} d x\right\}^{1 / q} \\
\equiv \mathrm{I}+\mathrm{II}, &
\end{aligned}
$$

where in the second step, we used Minkowski's inequality when $q \in(1, \infty)$; and when $q \in[p, 1]$, we used the fact that $(a+b)^{t} \leq \max \left\{2^{t-1}, 1\right\}\left(a^{t}+b^{t}\right)$ for all $a, b, t \in(0, \infty)$.

The estimate for II is easier. If $\phi_{\nu} * \psi_{j} * \varphi_{j} * f$ is a non-zero function, then using the fact that $\phi$ and $\varphi$ satisfy respectively (2.1) and Lemma 3.1(iv), we obtain $|j-\nu| \leq 2$. Therefore,

$$
\mathrm{II} \lesssim\left\{\sum_{|\nu|>N-2} \sum_{j=\nu-2}^{\nu+2} 2^{\nu s q} \int_{\mathbb{R}^{n}}\left|\left(\phi_{\nu} * \psi_{j} * \varphi_{j} * f\right)(x)\right|^{q} d x\right\}^{1 / q} .
$$

For the sake of simplicity, we only give the estimate for the $j=\nu$ term in the above formula, since the estimates for the other four terms are similar.

Recall that $f \in C_{c}^{\infty}\left(\mathbb{R}^{n}\right)$ has vanishing moments up to order $\mathcal{N}$ (if $\mathcal{N}=-1$, then $f$ has no vanishing moment), $\psi \in \mathcal{S}_{L_{0}}\left(\mathbb{R}^{n}\right), \phi \in \mathcal{S}_{\infty}\left(\mathbb{R}^{n}\right)$ and $\varphi \in \mathcal{S}_{\infty}\left(\mathbb{R}^{n}\right)$. For any given $M_{3}>\max \{n, n / q\}, L_{1} \in \mathbb{Z}_{+}$and $M_{1}>$ $\max \{n, n / q\}$, by Corollary 3.1(i) and Lemma 3.3(i) we find that for all $\nu \geq 0$ 
we have

$$
\begin{aligned}
\mid\left(\phi_{\nu} * \psi_{\nu}\right. & \left.* \varphi_{\nu} * f\right)(x) \mid \\
& \leq \int_{\mathbb{R}^{n}}\left|\left(\phi_{\nu} * \psi_{\nu}\right)(x-y)\right|\left|\left(\varphi_{\nu} * f\right)(y)\right| d y \\
& \lesssim \int_{\mathbb{R}^{n}} 2^{\nu n}\left(1+2^{\nu}|x-y|\right)^{-M_{3}} 2^{-\nu L_{1}}(1+|y|)^{-M_{1}} d y \\
& \sim \int_{|x| \geq 2|y|} 2^{\nu n}\left(1+2^{\nu}|x-y|\right)^{-M_{3}} 2^{-\nu L_{1}}(1+|y|)^{-M_{1}} d y \\
& \quad+\int_{|x|<2|y|} \cdots \\
& \lesssim 2^{\nu\left(n-L_{1}\right)}\left(1+2^{\nu}|x|\right)^{-M_{3}}+2^{-\nu L_{1}}(1+|x|)^{-M_{1}} .
\end{aligned}
$$

Applying Corollary 3.1(i) with $M_{3}>\max \{n, n / q\}$, Lemma 3.3(ii) with $L_{2}=$ $\mathcal{N}$ and $M_{2}>\max \{n, n / q\}$, we similarly infer that for all $\nu<0$,

$$
\begin{aligned}
& \left|\left(\phi_{\nu} * \psi_{\nu} * \varphi_{\nu} * f\right)(x)\right| \\
& \quad \lesssim 2^{\nu(n+\mathcal{N}+1)}\left(1+2^{\nu}|x|\right)^{-M_{3}}+2^{\nu(n+\mathcal{N}+1)}\left(1+2^{\nu}|x|\right)^{-M_{2}} .
\end{aligned}
$$

We may as well assume that $N>2$. Using (3.13) and (3.14), we then have $\left\{\sum_{|\nu|>N-2} 2^{\nu s q} \int_{\mathbb{R}^{n}}\left|\left(\phi_{\nu} * \psi_{\nu} * \varphi_{\nu} * f\right)(x)\right|^{q} d x\right\}^{1 / q}$

$\lesssim\left\{\sum_{\nu>N-2}\left[2^{\nu q\left(s+n-L_{1}-n / q\right)}+2^{\nu q\left(s-L_{1}\right)}\right]\right\}^{1 / q}+\left\{\sum_{\nu<-(N-2)} 2^{\nu q(s+n+\mathcal{N}+1-n / q)}\right\}^{1 / q}$.

We choose $L_{1}$ large enough satisfying

$$
\max \left\{s+n-L_{1}-n / q, s-L_{1}\right\}<n(1 / q-1 / p)-1 .
$$

Recalling that $\mathcal{N}=\max \{\lfloor n(1 / p-1)-s\rfloor,-1\}$, we then have

$$
s+n+\mathcal{N}+1-n / q>n(1 / p-1 / q) \geq 0 .
$$

Now we let

$$
\sigma \equiv s+n+\mathcal{N}+1-n / p
$$

Then $0<\sigma \leq \max \{s+n-n / p, 1\}$ and

$$
\left\{\sum_{|\nu| \geq N-2} 2^{\nu s q} \int_{\mathbb{R}^{n}}\left|\left(\phi_{\nu} * \psi_{\nu} * \varphi_{\nu} * f\right)(x)\right|^{q} d x\right\}^{1 / q} \lesssim\left(2^{-N}+2^{-N \sigma}\right) 2^{N n(1 / q-1 / p)}
$$

which implies that II has the desired estimate of (3.9).

We estimate I by considering the following two cases: $q \in(1, \infty)$ and $q \in[p, 1]$. 
CASE 1: $q \in(1, \infty)$. In this case, by Minkowski's inequality, we have

$$
\begin{aligned}
\mathrm{I} \leq & \sum_{|j| \leq N}\left\{\int_{\mathbb{R}^{n} \backslash Q\left(0,2^{N}\right)}\left|\left(\varphi_{j} * f\right)(y)\right| d y\right\} \\
& \times\left\{\sum_{\nu \in \mathbb{Z}} 2^{\nu s q} \int_{\mathbb{R}^{n}}\left|\left(\phi_{\nu} * \psi_{j}\right)(x)\right|^{q} d x\right\}^{1 / q} .
\end{aligned}
$$

Denote by $\mathcal{J}$ the integral in the first bracket of (3.18) and by $\mathcal{K}$ the summation in the second bracket of (3.18). For any given $L_{1} \in \mathbb{Z}_{+}$and $M_{1}>n$, using Lemma 3.3(i), we find that for all $j \geq 0$,

$$
\mathcal{J} \lesssim \int_{\mathbb{R}^{n} \backslash Q\left(0,2^{N}\right)} 2^{-j L_{1}}(1+|y|)^{-M_{1}} d y \lesssim 2^{-j L_{1}} 2^{-N\left(M_{1}-n\right)} .
$$

For any given $M_{2}>n$, using Lemma 3.3(ii) with $L_{2}=\mathcal{N}$, we deduce that for all $j<0$,

$$
\begin{aligned}
\mathcal{J} & \lesssim \int_{\mathbb{R}^{n} \backslash Q\left(0,2^{N}\right)} 2^{j(n+\mathcal{N}+1)}\left(1+2^{j}|y|\right)^{-M_{2}} d y \\
& \lesssim 2^{j(n+\mathcal{N}+1)-j M_{2}} 2^{-N\left(M_{2}-n\right)} .
\end{aligned}
$$

Recall that $L_{0} \in \mathcal{S}_{L_{0}}\left(\mathbb{R}^{n}\right)$. Then for any given $L_{3} \leq L_{0}, L_{4} \in \mathbb{Z}_{+}, M_{3}>n / q$ and $M_{4}>n / q$, applying Corollary 3.1(i), (ii), we find that for all $j \in \mathbb{Z}$,

$$
\mathcal{K} \lesssim \sum_{\nu \leq j} 2^{\nu s q+\nu n q-(j-\nu) L_{3} q-\nu n}+\sum_{\nu>j} 2^{\nu s q+\nu n q-(\nu-j)\left(n+L_{4}+1\right) q-j n} .
$$

Using (3.18)-(3.21) and the fact that for all $\kappa \in(0,1]$ and $a_{j} \in \mathbb{C}$,

$$
\left\{\sum_{j \in \mathbb{N}}\left|a_{j}\right|\right\}^{\kappa} \leq \sum_{j \in \mathbb{N}}\left|a_{j}\right|^{\kappa}
$$

and choosing $L_{1}, L_{3}, L_{4}, M_{1}$ and $M_{2}$ large enough so that $L_{1}>n+s-n / q$, $L_{3}>-n-s+n / q, L_{4}>s-1, M_{1}>n+1+n(1 / p-1 / q)$ and $M_{2}>$ $\mathcal{N}+2 n+s+1-n / q$, we obtain $I \lesssim\left(2^{-N}+2^{-N \sigma}\right) 2^{N n(1 / q-1 / p)}$ with $\sigma>0$ as in (3.17). This gives the desired estimate of I for $q \in(1, \infty)$.

CASE 2: $q \in[p, 1]$. In this case, we write

$$
\begin{aligned}
\sum_{|j| \leq N} & \int_{\mathbb{R}^{n} \backslash Q\left(0,2^{N}\right)}\left(\phi_{\nu} * \psi_{j}\right)(x-y)\left(\varphi_{j} * f\right)(y) d y \\
= & \sum_{0 \leq j \leq N, j \geq \nu} \int_{\mathbb{R}^{n} \backslash Q\left(0,2^{N}\right)}\left(\phi_{\nu} * \psi_{j}\right)(x-y)\left(\varphi_{j} * f\right)(y) d y \\
& +\sum_{\substack{0 \leq j \leq N, j<\nu \\
\cdots+}} \cdots+\sum_{-N \leq j<0, j \geq \nu} \cdots+\sum_{-N \leq j<0, j<\nu} \cdots \\
\equiv & \mathcal{J}_{1}(x)+\mathcal{J}_{2}(x)+\mathcal{J}_{3}(x)+\mathcal{J}_{4}(x) .
\end{aligned}
$$


Combining the expression of I with (3.22) and (3.23) yields

$$
\mathrm{I}^{q} \lesssim \sum_{\nu \in \mathbb{Z}} 2^{\nu s q} \int_{\mathbb{R}^{n}}\left(\left[\mathcal{J}_{1}(x)\right]^{q}+\left[\mathcal{J}_{2}(x)\right]^{q}+\left[\mathcal{J}_{3}(x)\right]^{q}+\left[\mathcal{J}_{4}(x)\right]^{q}\right) d x .
$$

To estimate $\mathcal{J}_{1}(x)$, we split the integral into two parts as in (3.13) and then use Lemma 3.3(i) and Corollary 3.1(i). Therefore, for any given $L_{1} \in \mathbb{Z}_{+}$, $M_{1}>2 n, L_{3} \leq L_{0}$ and $M_{3} \in \mathbb{Z}_{+}$, we have

$$
\begin{aligned}
& \mathcal{J}_{1}(x) \lesssim \sum_{0 \leq j \leq N, j \geq \nu} \int_{\mathbb{R}^{n} \backslash Q\left(0,2^{N}\right)} 2^{\nu n-(j-\nu) L_{3}-j L_{1}}\left(1+2^{\nu}|x-y|\right)^{-M_{3}}(1+|y|)^{-M_{1}} d y \\
& \lesssim \sum_{0 \leq j \leq N, j \geq \nu} 2^{\nu n-(j-\nu) L_{3}-j L_{1}}\left(1+2^{\nu}|x|\right)^{-M_{3}} \int_{\substack{y \notin Q\left(0,2^{N}\right) \\
|x| \geq 2|y|}}(1+|y|)^{-M_{1}} d y \\
& +\sum_{0 \leq j \leq N, j \geq \nu} 2^{\nu n-(j-\nu) L_{3}-j L_{1}}(1+|x|)^{-M_{1} / 2} \int_{\substack{y \notin Q\left(0,2^{N}\right) \\
|x| \geq 2|y|}}(1+|y|)^{-M_{1} / 2} d y \\
& \lesssim 2^{-N\left(M_{1}-n\right)} \sum_{0 \leq j \leq N, j \geq \nu} 2^{\nu n-(j-\nu) L_{3}-j L_{1}}\left(1+2^{\nu}|x|\right)^{-M_{3}} \\
& +2^{-N\left(M_{1} / 2-n\right)} \sum_{0 \leq j \leq N, j \geq \nu} 2^{\nu n-(j-\nu) L_{3}-j L_{1}}(1+|x|)^{-M_{1} / 2} .
\end{aligned}
$$

This combined with the fact $q \in[p, 1)$ and (3.22) implies that if we choose $L_{1}>n+s, L_{3}>-n-s+n / q, M_{1}>2 \max \{n / q, n(1 / p-1 / q)+n+1\}$ and $M_{3}>n / q$, then

$$
\begin{aligned}
\sum_{\nu \in \mathbb{Z}} 2^{\nu s q} \int_{\mathbb{R}^{n}}\left[\mathcal{J}_{1}(x)\right]^{q} d x & \lesssim 2^{-N q\left(M_{1}-n\right)}+2^{-N q\left(M_{1} / 2-n\right)} \\
& \lesssim 2^{-N q} 2^{N n q(1 / q-1 / p)} .
\end{aligned}
$$

For any given $L_{1} \in \mathbb{Z}_{+}, M_{1}>2 n, L_{4} \in \mathbb{Z}_{+}$and $M_{4} \in \mathbb{Z}_{+}$, by Lemma 3.3(i), Corollary 3.1(ii) and an argument similar to the estimate of $\mathcal{J}_{1}(x)$, we obtain

$$
\begin{aligned}
\mathcal{J}_{2}(x) \lesssim & \sum_{0 \leq j \leq N, j<\nu} 2^{\nu n-(\nu-j)\left(n+L_{4}+1\right)-j L_{1}} \\
& \times \int_{\mathbb{R}^{n} \backslash Q\left(0,2^{N}\right)}\left(1+2^{j}|x-y|\right)^{-M_{4}}(1+|y|)^{-M_{1}} d y \\
\lesssim & 2^{-N\left(M_{1}-n\right)} \sum_{0 \leq j \leq N, j<\nu} 2^{\nu n-(\nu-j)\left(n+L_{4}+1\right)-j L_{1}}\left(1+2^{j}|x|\right)^{-M_{4}} \\
& +2^{-N\left(M_{1} / 2-n\right)} \sum_{0 \leq j \leq N, j<\nu} 2^{\nu n-(\nu-j)\left(n+L_{4}+1\right)-j L_{1}}(1+|x|)^{-M_{1} / 2} .
\end{aligned}
$$

We choose $L_{4}>s-1$ and $L_{1}>n+s$. From this, the estimate for $\mathcal{J}_{2}(x)$, (3.22) and the assumption $M_{1}>2 \max \{n / q, n(1 / p-1 / q)+n+1\}$, it follows 
that

$$
\begin{aligned}
\sum_{\nu \in \mathbb{Z}} 2^{\nu s q} \int_{\mathbb{R}^{n}}\left[\mathcal{J}_{2}(x)\right]^{q} d x & \lesssim 2^{-N q\left(M_{1}-n\right)}+2^{-N q\left(M_{1} / 2-n\right)} \\
& \lesssim 2^{-N q} 2^{N n q(1 / q-1 / p)}
\end{aligned}
$$

For any given $L_{3} \leq L_{0}, M_{2}>2 n$ and $M_{3} \in \mathbb{Z}_{+}$, applying Lemma 3.3(ii) and Corollary 3.1(i) yields

$$
\begin{aligned}
\mathcal{J}_{3}(x) \lesssim & \sum_{-N \leq j<0, j \geq \nu} 2^{\nu n-(j-\nu) L_{3}+j(n+\mathcal{N}+1)} \\
& \times \int_{\mathbb{R}^{n} \backslash Q\left(0,2^{N}\right)}\left(1+2^{\nu}|x-y|\right)^{-M_{3}}\left(1+2^{j}|y|\right)^{-M_{2}} d y \\
\lesssim & 2^{-N\left(M_{2}-n\right)} \sum_{-N \leq j<0, j \geq \nu} 2^{\nu n-(j-\nu) L_{3}+j(n+\mathcal{N}+1)-j M_{2}}\left(1+2^{\nu}|x|\right)^{-M_{3}} \\
& +2^{-N\left(M_{2} / 2-n\right)} \sum_{-N \leq j<0, j \geq \nu} 2^{\nu n-(j-\nu) L_{3}+j(n+\mathcal{N}+1)-j M_{2} / 2}\left(1+2^{j}|x|\right)^{-M_{2} / 2}
\end{aligned}
$$

We choose $M_{2}>2 \max \{n / q, s+2 n+1+\mathcal{N}-n / q\}$ and $L_{3}>-n-s+n / q$. From this, (3.22) and (3.16), we conclude that for $\sigma$ as in (3.17),

$$
\sum_{\nu \in \mathbb{Z}} 2^{\nu s q} \int_{\mathbb{R}^{n}}\left[\mathcal{J}_{3}(x)\right]^{q} d x \lesssim 2^{-N q\left(M_{2} / 2-n\right)} \lesssim 2^{-N \sigma q} 2^{-N n q(1 / q-1 / p)} .
$$

Now we estimate $\mathcal{J}_{4}(x)$. For any given $M_{2}, M_{4}, L_{4} \in \mathbb{Z}_{+}$, using Lemma 3.3(ii) and Corollary 3.1(ii), we have

$$
\begin{aligned}
\mathcal{J}_{4}(x) \lesssim & \sum_{-N \leq j<0, j<\nu} 2^{\nu n-(\nu-j)\left(n+L_{4}+1\right)+j(n+\mathcal{N}+1)} \\
& \times \int_{\mathbb{R}^{n} \backslash Q\left(0,2^{N}\right)}\left(1+2^{j}|x-y|\right)^{-M_{4}}\left(1+2^{j}|y|\right)^{-M_{2}} d y \\
\lesssim & 2^{-N\left(M_{2}-n\right)} \sum_{-N \leq j<0, j<\nu} 2^{\nu n-(\nu-j)\left(n+L_{4}+1\right)+j(n+\mathcal{N}+1)-j M_{2}}\left(1+2^{j}|x|\right)^{-M_{4}} \\
& +2^{-N\left(M_{2} / 2-n\right)} \sum_{-N \leq j<0, j<\nu} 2^{\nu n-(\nu-j)\left(n+L_{4}+1\right)+j(n+\mathcal{N}+1)-j M_{2} / 2} \\
& \times\left(1+2^{j}|x|\right)^{-M_{2} / 2} .
\end{aligned}
$$

Choose $M_{2}>2 \max \{n / q, s+2 n+1+\mathcal{N}-n / q\}$ and $L_{4}>s-1$. From this and the estimate of $\mathcal{J}_{4}(x)$ together with (3.22) and (3.16), it follows that for $\sigma$ as in (3.17),

$$
\sum_{\nu \in \mathbb{Z}} 2^{\nu s q} \int_{\mathbb{R}^{n}}\left[\mathcal{J}_{4}(x)\right]^{q} d x \lesssim 2^{-N q\left(M_{2} / 2-n\right)} \lesssim 2^{-N \sigma q} 2^{-N n q(1 / q-1 / p)} .
$$

Combining (3.23) through (3.28) yields the desired estimate of I for $q$ in 
$[p, 1)$. This combined with the argument of Case 1 and the estimate of II implies the validity of (3.9) for all $q \in[p, \infty)$, which completes the proof of Theorem 2.1.

REMARK 3.1. From the proof of Theorem 2.1, it follows that we need to assume that $\psi \in \mathcal{S}_{L_{0}}\left(\mathbb{R}^{n}\right)$ with $L_{0} \in \mathbb{Z}_{+}$and $L_{0}>-n-s+n / q$.

4. Proof of Theorem 2.2. Applying Theorem 2.1 and following a standard argument, we can obtain Theorem 2.2. To this end, we need the following density lemma.

Lemma 4.1. Let $s \in \mathbb{R}, p \in(0,1]$ and $q \in[p, \infty)$. Then $\dot{F}_{p, q}^{s, \text { fin }}\left(\mathbb{R}^{n}\right)$ is dense in $\dot{F}_{p, q}^{s}\left(\mathbb{R}^{n}\right)$.

Proof. Fix $f \in \dot{F}_{p, q}^{s}\left(\mathbb{R}^{n}\right)$. It suffices to show that for any given $\varepsilon>0$, there exists $h \in \dot{F}_{p, q}^{s, f i n}\left(\mathbb{R}^{n}\right)$ such that $\|f-h\|_{\dot{F}_{p, q}^{s}\left(\mathbb{R}^{n}\right)}<\varepsilon$.

Since $\mathcal{S}_{\infty}\left(\mathbb{R}^{n}\right) \subset \dot{F}_{p, q}^{s}\left(\mathbb{R}^{n}\right)$ and $\mathcal{S}_{\infty}\left(\mathbb{R}^{n}\right)$ is a dense subset of $\dot{F}_{p, q}^{s}\left(\mathbb{R}^{n}\right)$ (see [23, p. 240]), it follows that there exists some $g \in \mathcal{S}_{\infty}\left(\mathbb{R}^{n}\right)$ such that $\|g-f\|_{\dot{F}_{p, q}^{s}\left(\mathbb{R}^{n}\right)}<\varepsilon / 3$.

Applying Lemma 3.2, we find that $g=\sum_{k \in \mathbb{Z}} \psi_{k} * \varphi_{k} * g$ in $\mathcal{S}_{\infty}\left(\mathbb{R}^{n}\right)$, where $\psi$ and $\varphi$ are as in Lemma 3.2. For any $N \in \mathbb{N}$, set

$$
g_{N} \equiv \sum_{|k| \leq N} \psi_{k} * \varphi_{k} * g
$$

Then there exists $N \in \mathbb{N}$ large enough such that $\left\|g_{N}-g\right\|_{\dot{F}_{p, q}^{s}\left(\mathbb{R}^{n}\right)}<\varepsilon / 3$ (see $[23$, p. 240] again). Now we use the same notation as in the proof of Theorem 2.1. For any $k \in \mathbb{Z}$, set $\Omega_{k} \equiv\left\{x \in \mathbb{R}^{n}: \dot{S}_{a, q}^{s}(g)(x)>2^{k}\right\}$ and

$$
\mathcal{Q}_{k} \equiv\left\{Q \in \mathcal{Q}:\left|Q \cap \Omega_{k}\right|>|Q| / 2,\left|Q \cap \Omega_{k+1}\right| \leq|Q| / 2\right\} .
$$

Denote by $\left\{Q_{k}^{i}\right\}_{i \in I_{k}}$ the collection of all maximal dyadic cubes in $\mathcal{Q}_{k}$, where $I_{k}$ is the index set. Similarly to (3.3), we write $g_{N}$ as

$$
\begin{aligned}
g_{N}(x) & =\sum_{|k| \leq N} \sum_{\left\{Q \in \mathcal{Q}: \ell(Q)=2^{-k}\right\}} \int_{Q} \psi_{k}(x-y)\left(\varphi_{k} * g\right)(y) d y \\
& =\sum_{\left\{Q \in \mathcal{Q}: 2^{-N} \leq \ell(Q) \leq 2^{N}\right\}} \int_{Q} \psi_{Q}(x-y)\left(\varphi_{Q} * g\right)(y) d y \\
& =\sum_{k \in \mathbb{Z}} \sum_{i \in I_{k}}\left\{\sum_{\substack{Q \subset Q_{k}^{i}, Q \in \mathcal{Q}_{k} \\
2^{-N} \leq \ell(Q) \leq 2^{N}}} \int_{Q} \psi_{Q}(x-y)\left(\varphi_{Q} * g\right)(y) d y\right\} \\
& \equiv \sum_{k \in \mathbb{Z}} \sum_{i \in I_{k}} \mu_{k, i} b_{k, i},
\end{aligned}
$$


where $\mu_{k, i}$ and $b_{k, i}$ are defined as $\lambda_{k, i}$ and $a_{k, i}$ in the proof of Theorem 2.1 with $\sum_{Q \subset Q_{k}^{i}, Q \in \mathcal{Q}_{k}}$ replaced by $\sum_{\left\{Q: Q \subset Q_{k}^{i}, Q \in \mathcal{Q}_{k}, 2^{-N} \leq \ell(Q) \leq 2^{N}\right\}}$. Arguing as in the proof of [16, Theorem 1], we conclude that each $b_{k, i}$ is a $(p, q, s)$-atom of $\dot{F}_{p, q}^{s}\left(\mathbb{R}^{n}\right)$ and

$$
\left\{\sum_{k \in \mathbb{Z}} \sum_{i \in I_{k}}\left|\mu_{k, i}\right|^{p}\right\}^{1 / p} \lesssim\|g\|_{\dot{F}_{p, q}^{s}\left(\mathbb{R}^{n}\right)}<\infty
$$

It is easy to see that the series in the bracket of (4.1) belongs to $C_{c}^{\infty}\left(\mathbb{R}^{n}\right)$ since it has only finitely many terms. This implies that each $b_{k, i}$ is in $C_{c}^{\infty}\left(\mathbb{R}^{n}\right)$.

For any $M \in \mathbb{N}$, we set $h_{M}^{N} \equiv \sum_{|k| \leq M} \sum_{i \in I_{k}, i \leq M} \mu_{k, i} b_{k, i}$. Then there exists $M \in \mathbb{N}$ large enough such that $\left\|h_{M}^{N}-g_{N}\right\|_{\dot{F}_{p, q}^{s}\left(\mathbb{R}^{n}\right)}<\varepsilon / 3$. Therefore,

$$
\begin{aligned}
\left\|h_{M}^{N}-f\right\|_{\dot{F}_{p, q}^{s}\left(\mathbb{R}^{n}\right)}^{p} & \leq\left\|h_{M}^{N}-g_{N}\right\|_{\dot{F}_{p, q}^{s}\left(\mathbb{R}^{n}\right)}^{p}+\left\|g_{N}-g\right\|_{\dot{F}_{p, q}^{s}\left(\mathbb{R}^{n}\right)}^{p}+\|g-f\|_{\dot{F}_{p, q}^{s}\left(\mathbb{R}^{n}\right)} \\
& <\varepsilon .
\end{aligned}
$$

Setting $h \equiv h_{M}^{N}$ completes the proof of Lemma 4.1.

Proof of Theorem 2.2. For any given $f \in \dot{F}_{p, q}^{s, \text { fin }}\left(\mathbb{R}^{n}\right)$, by Theorem 2.1, we write $f=\sum_{j=1}^{N} \lambda_{j} a_{j}$, where $N \in \mathbb{N},\left\{\lambda_{j}\right\}_{j=1}^{N} \subset \mathbb{C},\left\{a_{j}\right\}_{j=1}^{N}$ are infinitely differentiable $(p, q, s)$-atoms of $\dot{F}_{p, q}^{s}\left(\mathbb{R}^{n}\right)$, and $\sum_{j=1}^{N}\left|\lambda_{j}\right|^{p} \lesssim\|f\|_{\dot{F}_{p, q}^{s}\left(\mathbb{R}^{n}\right)}^{p}$. Using the assumption $r \in[p, 1]$ together with (2.7), (2.9) and (3.22), we obtain

$$
\|T(f)\|_{\mathcal{B}_{r}} \lesssim\left\{\sum_{j=1}^{N}\left|\lambda_{j}\right|^{r}\right\}^{1 / r} \leq\left\{\sum_{j=1}^{N}\left|\lambda_{j}\right|^{p}\right\}^{1 / p}
$$

Taking the infimum over all finite atomic decompositions of $f$ and using Corollary 2.1, we see that for all $f \in \dot{F}_{p, q}^{s, \text { fin }}\left(\mathbb{R}^{n}\right)$,

$$
\|T(f)\|_{\mathcal{B}_{r}} \lesssim\|f\|_{\dot{F}_{p, q}^{s}\left(\mathbb{R}^{n}\right)} \text {. }
$$

For any given $f \in \dot{F}_{p, q}^{s}\left(\mathbb{R}^{n}\right)$, by Lemma 4.1 , there exists a sequence $\left\{f_{m}\right\}_{m=1}^{\infty} \subset \dot{F}_{p, q}^{s, \text { fin }}\left(\mathbb{R}^{n}\right)$ such that $\left\|f-f_{m}\right\|_{\dot{F}_{p, q}^{s}\left(\mathbb{R}^{n}\right)} \rightarrow 0$ as $m \rightarrow \infty$. This combined with (2.8) and (4.2) implies that $\left\{T\left(f_{m}\right)\right\}_{m=1}^{\infty}$ is a Cauchy sequence in $\mathcal{B}_{r}$. So we define $\widetilde{T}(f)=\lim _{m \rightarrow \infty} T\left(f_{m}\right)$, where the limit is taken in $\mathcal{B}_{r}$. It follows that $\widetilde{T}(f)$ is well defined, and $\widetilde{T}$ is bounded from $\dot{F}_{p, q}^{s}\left(\mathbb{R}^{n}\right)$ to $\mathcal{B}_{r}$.

Supposed that $\widetilde{T}^{\prime}$ is another bounded extension of $T$. That is, $\widetilde{T}^{\prime}$ is bounded from $\dot{F}_{p, q}^{s}\left(\mathbb{R}^{n}\right)$ to $\mathcal{B}_{r}$, and $\widetilde{T}^{\prime}(f)=T(f)$ for all $f \in \dot{F}_{p, q}^{s, \text { fin }}\left(\mathbb{R}^{n}\right)$. From this and (2.8), we conclude that for any $f \in \dot{F}_{p, q}^{s}\left(\mathbb{R}^{n}\right)$,

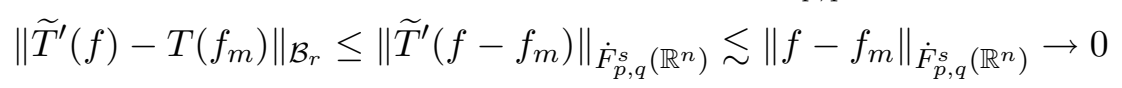

as $m \rightarrow \infty$. This implies that $\widetilde{T}^{\prime}=\widetilde{T}$.

Therefore, $\widetilde{T}$ is the unique bounded extension of $T$, which completes the proof of Theorem 2.2 . 


\section{References}

[1] T. Aoki, Locally bounded linear topological spaces, Proc. Imp. Acad. Tokyo 18 (1942), 588-594.

[2] M. Bownik, Boundedness of operators on Hardy spaces via atomic decompositions, Proc. Amer. Math. Soc. 133 (2005), 3535-3542.

[3] M. Bownik, B. Li, D. Yang and Y. Zhou, Weighted anisotropic product Hardy spaces and their applications in boundedness of sublinear operators, in preparation.

[4] S. Y. A. Chang and R. Fefferman, A continuous version of duality of $H^{1}$ with BMO on the bidisc, Ann. of Math. 112 (1980), 179-201.

[5] D. Chen, J. Chen and D. Fan, Rough singular integral operators on Hardy-Sobolev spaces, Appl. Math. J. Chinese Univ. Ser. B 20 (2005), 1-9.

[6] J. Chen, D. Fan and Y. Ying, Certain operators with rough singular kernels, Canad. J. Math. 55 (2003), 504-532.

[7] R. R. Coifman and G. Weiss, Analyse Harmonique Non-commutative sur Certains Espaces Homogènes, Lecture Notes in Math. 242, Springer, Berlin, 1971.

[8] - - - Extensions of Hardy spaces and their use in analysis, Bull. Amer. Math. Soc. 83 (1977), 569-645.

[9] M. Frazier and B. Jawerth, Decomposition of Besov spaces, Indiana Univ. Math. J. 34 (1985), 777-799.

[10] - , - A discrete transform and decompositions of distribution spaces, J. Funct. Anal. 93 (1990), 34-170.

[11] M. Frazier, B. Jawerth and G. Weiss, Littlewood-Paley Theory and the Study of Function Spaces, CBMS Reg. Conf. Ser. Math. 79. Amer. Math. Soc., Providence, RI, 1991.

[12] J. García-Cuerva and J. L. Rubio de Francia, Weighted Norm Inequalities and Related Topics, North-Holland, Amsterdam, 1985.

[13] L. Grafakos, L. Liu and D. Yang, Maximal function characterizations of Hardy spaces on RD-spaces and their applications, Sci. China (Ser. A), to appear.

[14] Y. Han, D. Müller and D. Yang, A theory of Besov and Triebel-Lizorkin spaces on metric measure spaces modeled on Carnot-Carathéodory spaces, Abstr. Appl. Anal. 2008, Art. ID 893409, to appear.

[15] - , 一, 一, Littlewood-Paley characterizations for Hardy spaces on spaces of homogeneous type, Math. Nachr. 279 (2006), 1505-1537.

[16] Y. Han, M. Paluszyński and G. Weiss, A new atomic decomposition for the TriebelLizorkin spaces, in: Harmonic Analysis and Operator Theory (Caracas, 1994), Contemp. Math. 189, Amer. Math. Soc., Providence, RI, 1995, 235-249.

[17] G. Hu, D. Yang and Y. Zhou, Boundedness of singular integrals in Hardy spaces on spaces of homogeneous type, Taiwanese J. Math., to appear.

[18] K.-S. Lau and L. Yan, Wavelet decomposition of Calderón-Zygmund operators on function spaces, J. Austral. Math. Soc. 77 (2004), 29-46.

[19] S. Meda, P. Sjögren and M. Vallarino, On the $H^{1}-L^{1}$ boundedness of operators, Proc. Amer. Math. Soc. 136 (2008), 2921-2931.

[20] Y. Meyer and R. Coifman, Wavelets. Calderón-Zygmund and Multilinear Operators, Cambridge Univ. Press, Cambridge, 1997.

[21] Y. Meyer, M. Taibleson and G. Weiss, Some functional analytic properties of the spaces $B_{q}$ generated by blocks, Indiana Univ. Math. J. 34 (1985), 493-515.

[22] S. Rolewicz, Metric Linear Spaces, 2nd ed., Math. Appl. (East Eur. Ser.) 20, Reidel, Dordrecht, and PWN, Warszawa, 1985.

[23] H. Triebel, Theory of Function Spaces, Birkhäuser, Basel, 1983. 
[24] K. Yabuta, A remark on the $\left(H^{1}, L^{1}\right)$ boundedness, Bull. Fac. Sci. Ibaraki Univ. Ser. A 25 (1993), 19-21.

[25] D. Yang and Y. Zhou, A bounded criterion via atoms for linear operators in Hardy spaces, Constr. Approx., to appear.

[26] - - - Boundedness of sublinear operators in Hardy spaces on RD-spaces via atoms, J. Math. Anal. Appl. 339 (2008), 622-635.

[27] K. Zhao, Boundedness of operators on Hardy spaces, Taiwanese J. Math., to appear.

School of Mathematical Sciences

Beijing Normal University

Laboratory of Mathematics and Complex Systems

Ministry of Education

Beijing 100875

People's Republic of China

E-mail: liuliguang@mail.bnu.edu.cn

dcyang@bnu.edu.cn

Received December 4, 2007

Revised version July 30, 2008 